\title{
Modeling and Imaging Techniques with Potential for Application in Bioterrorism
}

\author{
H.T. Banks ${ }^{1}$, David Bortz ${ }^{2}$, Gabriella Pinter ${ }^{3}$ and Laura Potter ${ }^{1,4}$ \\ ${ }^{1}$ Center for Research in Scientific Computation, North Carolina State \\ University, Raleigh, NC 27695-8205; ${ }^{2}$ Department of Mathematics, \\ University of Michigan, Ann Arbor, MI 48109; ${ }^{3}$ Department of \\ Mathematical Sciences, University of Wisconsin-Milwaukee, \\ Milwaukee, WI 53201
}

\section{Introduction}

In this paper we present a survey of several recent and emerging ideas and efforts on modeling and system interrogation in the presence of uncertainty that we feel have significant potential for applications related to bioterrorism. The first focuses on physiologically based pharmacokinetic (PBPK) type models and the effects of drugs, toxins and viruses on tissue, organs, individuals and populations wherein both intra- and inter-individual variability are present when one attempts to determine kinetic rates, susceptibility, efficacy of toxins, antitoxins, etc., in aggregate populations. Methods combining deterministic and stochastic concepts are necessary to formulate and computationally solve the associated estimation problems. Similar issues arise in the HIV infectious models we also present below.

A second effort concerns the use of remote electromagnetic interrogation pulses linked to dielectric properties of materials to carry out macroscopic structural imaging of bulk packages (drugs, explosives, etc.) as well as test for presence and levels of toxic chemical compounds in tissue. These techniques also may be useful in functional imaging (e.g., of brain and CNS activity levels) to determine levels of threat in potential adversaries via changes in dielectric properties and conductivity.

The PBPK and cellular level virus infectious models we discuss are special examples of a much wider class of population models that one might utilize to investigate potential agents for use in attacks, such as viruses, bacteria, fungi and other chemical, biochemical or radiological agents. These include general epidemiological models such as SIR infectious

\footnotetext{
${ }^{4}$ Current address: Scientific Computing and Mathematical Modeling, GlaxoSmithKline, Research Triangle Park, NC 27709.
} 
spread models containing contact and susceptibility rates with structures (e.g., public vs. private transport; residence times in exposure; subnetworks of populations) as well as more general population models with heterogeneities and/or behavioral structures (e.g., social interaction, age/size dependency, spatial/temporal dependency, adaptive transient behavior, etc.). These may involve general dynamical systems, both discrete and continuous, including ordinary and/or partial differential equations and delay differential equations. Included are well known structured population models, such as those of Sinko-Streifer and McKendrickVon Foerster. These deterministic models often must be augmented with probabilistic and/or statistical structures such as mixing distributions, random effects, etc. (see [20, 22] for discussions and references). Such models combine ideas from continuum population models with aspects of agent based models incorporating individual level effects. The results are population models encompassing intra-individual and/or inter-individual variability that in some cases describe (predict) continuous population evolution that is driven by distributions of individual level mechanisms and behaviors. The models described in Section 2 below, where the parameters are viewed as random variables, or realizations thereof, are examples of these.

The use of models such as those outlined above ultimately lead to estimation or inverse problems containing both a mathematical model and a statistical model. These are treated in a fit-to-data formulation using either experimental data or synthetic "data" simulating a desired response. The latter arises, for example, in design of a drug or therapy that will result in a sought-after response of an individual or a population to a threat. However, the rationale to support elaborate models with structures does not lie simply in the desire to better fit a data set, but rather to aid in understanding basic mechanisms, pathways, behavior, etc. and to better frame population as well as individual responses to a challenge or to a prophylactic. But, it is not just inverse problems that arise in the context of these models (although that is the focus in this chapter); indeed, ideas from control theory and system optimization are also important. In almost every instance, including those discussed in the examples below, fundamental mathematics, especially modeling, theoretical and computational analysis, probability and statistics, play a significant role.

The electromagnetic interrogation and imaging ideas discussed in Section 3 could conceivably be a part of a surveillance technology in a first line of defense against bioterrorism. More precisely, physical detection and identification of hidden substances and agents (whether in food and water supplies, luggage, mail and packages, etc.) as a part of biodefense depends not only on the electromagnetic techniques discussed below, but also on characterization of dielectric properties of specific molecules and compounds. Although we present only deterministic aspects of the problems here, it can be expected that a successful methodology will also involve probabilistic and statistical formulations as well as tools from computational molecular biology. 


\section{Inverse Problems}

In these discussions we shall consider inverse or estimation problems involving aggregate data for populations which may be described by two different types of "parameter dependent" dynamics; for the lack of better terms we shall refer to these as "individual dynamics" and "aggregate dynamics". In both cases the data and populations inherently contain variability of parameters; this variability may be intra-individual, inter-individual or both.

The problems for individual dynamics can be used to treat many examples of practical interest including physiologically based pharmacokinetic (PBPK) models, biologically based dose response (BBDR) models, and susceptible-infectious-recovered (SIR) models of disease spread. The aggregate dynamics problems include cellular level virus models such as those for human immunodeficiency virus (HIV) growth.

In the first type of problem we consider below, one has a mathematical model at what we shall term (in perhaps something of a misnomer) the "individual" level. That is, the population count or density is described by a parameter dependent system. To facilitate our discussions here we use, without loss of generality, ordinary differential equation (ODE) models of the form

$$
\dot{x}(t)=f(t, x(t), q), \quad q \in Q,
$$

where the parameters $q$ (e.g., growth, mortality, fecundity, etc.) in the model vary from individual to individual across the population according to some probability distribution $P$ on a set of admissible parameters $Q$. More precisely, we suppose that the population is made up of subpopulations distinguished by common values of the parameters $q$ and whose time course is described by the solution $x(t ; q)$ of $(2.1)$ for the shared value of $q$. The total population count or density is then given by a weighted sum of these solutions over all possible $q \in Q$ so that the counts or densities one expects to observe at any time $t$ are given by

$$
\begin{aligned}
\bar{x}(t ; P) & =\mathcal{E}[x(t ; q) \mid P] \\
& \equiv \int_{Q} x(t ; q) d P(q)
\end{aligned}
$$

Experimental observations or data $\left\{\hat{d}_{i}\right\}$ corresponding to times $\left\{t_{i}\right\}$ are then given by the expected values $\bar{x}\left(t_{i} ; P\right)$ of $(2.2)$ plus some error $\varepsilon_{i}$ so that

$$
\hat{d}_{i}=\bar{x}\left(t_{i} ; P\right)+\varepsilon_{i}
$$

Assumptions about the error $\left\{\varepsilon_{i}\right\}$ in the observation process constitute the basis of an associated statistical model for the inverse problems. For discussions in this chapter, we will simply (and perhaps naively) assume that the errors are independent identically distributed (i.i.d.) Gaussian and will use an ordinary least squares (OLS) formulation for our inverse problems. This will then be completely equivalent to the traditional maximum likelihood 
estimator (MLE) problems. Thus, we formulate our inverse problem in terms of seeking to minimize

$$
J(P)=\sum_{i=1}^{n}\left|\mathcal{E}\left[x\left(t_{i} ; q\right) \mid P\right]-\hat{d}_{i}\right|^{2}
$$

over $P$ in the set $\mathcal{P}(Q)$ of probability measures on $Q$ subject to $t \rightarrow x(t ; q)$ satisfying $(2.1)$ for a given $q \in Q$.

The second type of problem involves aggregate dynamics wherein one has ODEs that describe the expected values of the population counts or densities. Essentially one has dynamics which already have been summed over the variability in parameters resulting in measure dependent dynamics (as opposed to parameter dependent dynamics) given by

$$
\dot{\bar{x}}(t)=g(t, \bar{x}(t), P), \quad P \in \mathcal{P}(Q),
$$

where now $\bar{x}(t ; P)$ is the average or expected value of the population count or density at time $t$. In this case the OLS formulation takes the form of minimizing

$$
J(P)=\sum_{i=1}^{n}\left|\bar{x}\left(t_{i} ; P\right)-\hat{d}_{i}\right|^{2}
$$

over $P \in \mathcal{P}(Q)$ subject to the aggregate dynamics (2.4). As we shall note in the examples below, models such as (2.4) occur naturally and may not be readily formulated in terms of dynamics of the form (2.1) and vice versa.

In Section 2.1 we outline a theoretical and computational framework for problems involving (2.1), (2.3) and illustrate the approach with a PBPK model for trichloroethylene (TCE). We follow this by discussing a framework for problems based on (2.4), (2.5) in the context of an inverse problem for virus dynamics (HIV in this case).

\subsection{Inverse Problems for Individual Dynamics}

Our goal is to estimate $q \in Q \subset R^{m}$ from solutions of $\dot{x}(t)=f(t, x(t), q)$. To do this we visualize parameters as realizations of a random variable and attempt to estimate the probability distribution function $(\mathrm{PDF}) P \in \mathcal{P}(Q)$ where $\mathcal{P}(Q)$ is the set of all PDFs on the Borel subsets of $Q$. We then attempt to estimate $P$ from given data $\hat{d}_{i}, i=1, \ldots, n$ where

$$
\begin{aligned}
\hat{d}_{i} & \approx \mathcal{E}\left[x\left(t_{i} ; q\right) \mid P\right] \\
& =\int_{Q} x\left(t_{i} ; q\right) d P(q),
\end{aligned}
$$

which in the case of a discrete probability measure can be written as

$$
\hat{d}_{i} \approx \sum_{j=1}^{M} x\left(t_{i}, q_{j}\right) p_{j}
$$


for $P$ a discrete PDF with atoms at $\left\{q_{j}\right\}_{j=1}^{M} \subset Q$ and associated probabilities $\left\{p_{j}\right\}_{j=1}^{M}$.

We can then, as noted above, define the OLS estimation problem of minimizing

$$
J(P)=\sum_{i=1}^{n}\left|\mathcal{E}\left[x\left(t_{i} ; q\right) \mid P\right]-\hat{d}_{i}\right|^{2}
$$

over $P \in \mathcal{P}(Q)$. To consider a theoretical and computational foundation for such problems, one needs the following items:

(i.) A topology on $\mathcal{P}(Q)$;

(ii.) Continuity of $P \rightarrow J(P)$;

(iii.) Compatible compactness results (well-posedness);

(iv.) Computational tools (approximations, etc.).

Fortunately, probability theory offers a great start toward a possible complete, tractable computational methodology [16]. The most important tool is the Prohorov metric, which we proceed to define. Suppose $(Q, d)$ is a complete metric space. For any closed subset $F \subset Q$ and $\varepsilon>0$, define

$$
F^{\varepsilon}=\{q \in Q: d(\tilde{q}, q)<\varepsilon, \tilde{q} \in F\}
$$

We then define the Prohorov metric $\rho: \mathcal{P}(Q) \times \mathcal{P}(Q) \rightarrow R^{+}$by

$$
\rho\left(P_{1}, P_{2}\right) \equiv \inf \left\{\varepsilon>0: P_{1}[F] \leq P_{2}\left[F^{\varepsilon}\right]+\varepsilon, F \text { closed, } F \subset Q\right\} \text {. }
$$

This can be shown to be a metric on $\mathcal{P}(Q)$ and has a number of well known properties including

(a.) $(\mathcal{P}(Q), \rho)$ is a complete metric space;

(b.) If $Q$ is compact, then $(\mathcal{P}(Q), \rho)$ is a compact metric space.

We note that the definition of $\rho$ is not intuitive. For example, what does $P_{k} \rightarrow P$ in $\rho$ mean? We have the following important characterizations [16].

Theorem 2.1 Given $P_{k}, P \in \mathcal{P}(Q)$, the following convergence statements are equivalent:

(i.) $\rho\left(P_{k}, P\right) \rightarrow 0$;

(ii.) $\int_{Q} f d P_{k}(q) \rightarrow \int_{Q} f d P(q)$ for all bounded, uniformly continuous $f: Q \rightarrow R^{1}$;

(iii.) $P_{k}[A] \rightarrow P[A]$ for all Borel sets $A \subset Q$ with $P[\partial A]=0$. 
Thus, one obtains immediately the following useful results:

- Convergence in the $\rho$ metric is equivalent to convergence in distribution;

- Let $C_{B}^{*}(Q)$ denote the topological dual of $C_{B}(Q)$, where $C_{B}(Q)$ is the usual space of bounded continuous functions on $Q$ with the supremum norm. If we view $\mathcal{P}(Q) \subset$ $C_{B}^{*}(Q)$, convergence in the $\rho$ topology is equivalent to weak* convergence in $\mathcal{P}(Q)$.

More importantly,

$$
\rho\left(P_{k}, P\right) \rightarrow 0 \text { is equivalent to } \int_{Q} x\left(t_{i} ; q\right) d P_{k}(q) \rightarrow \int_{Q} x\left(t_{i} ; q\right) d P(q),
$$

and $P_{k} \rightarrow P$ in $\rho$ metric is hence equivalent to

$$
\mathcal{E}\left[x\left(t_{i} ; q\right) \mid P_{k}\right] \rightarrow \mathcal{E}\left[x\left(t_{i} ; q\right) \mid P\right]
$$

or "convergence in expectation." This yields that

$$
P \rightarrow J(P)=\sum_{i=1}^{n}\left|\mathcal{E}\left[x\left(t_{i} ; q\right) \mid P\right]-\hat{d}_{i}\right|^{2}
$$

is continuous in the $\rho$ topology. Continuity of $P \rightarrow J(P)$ and compactness of $\mathcal{P}(Q)$ (each with respect to the $\rho$ metric) allows one to assert the existence of a solution to min $J(P)$ over $P \in \mathcal{P}(Q)$.

\subsubsection{Computational issues and approximation ideas}

We first note that $(\mathcal{P}(Q), \rho)$ is infinite-dimensional and hence one must use finite-dimensional approximations to obtain tractable computational algorithms. To this end, one may prove $($ see $[5])$

Theorem 2.2 Let $Q$ be a complete, separable metric space with metric $d, \mathcal{S}$ the class of all Borel subsets of $Q$ and $\mathcal{P}(Q)$ the space of probability measures on $(Q, \mathcal{S})$. Let $Q_{0}=\left\{q_{j}\right\}_{j=1}^{\infty}$ be a countable, dense subset of $Q$. Then the set of $P \in \mathcal{P}(Q)$ such that $P$ has finite support in $Q_{0}$ and rational masses is dense in $\mathcal{P}(Q)$ in the $\rho$ metric. That is,

$$
\mathcal{P}_{0}(Q) \equiv\left\{P \in \mathcal{P}(Q): P=\sum_{j=1}^{k} p_{j} \delta_{q_{j}}, k \in \mathcal{N}^{+}, q_{j} \in Q_{0}, p_{j} \text { rational, } \sum_{j=1}^{k} p_{j}=1\right\}
$$

is dense in $\mathcal{P}(Q)$ relative to $\rho$, where $\delta_{q_{j}}$ is the Dirac measure with atom at $q_{j}$. 
Given $Q_{d}=\bigcup_{M=1}^{\infty} Q_{M}$ with $Q_{M}=\left\{q_{j}^{M}\right\}_{j=1}^{M}$ chosen so that $Q_{d}$ is dense in $Q$, define

$$
\mathcal{P}^{M}(Q)=\left\{P \in \mathcal{P}(Q): P=\sum_{j=1}^{M} p_{j} \delta_{q_{j}^{M}}, q_{j}^{M} \in Q_{M}, p_{j} \text { rational, } \sum_{j=1}^{k} p_{j}=1\right\}
$$

Then we find

- $\mathcal{P}^{M}(Q)$ is a compact subset of $(\mathcal{P}(Q), \rho)$;

- $\mathcal{P}^{M}(Q) \not \subset \mathcal{P}^{M+1}(Q)$;

- " $\mathcal{P}^{M}(Q) \rightarrow \mathcal{P}(Q)$ " in the $\rho$ topology; that is, elements in $\mathcal{P}(Q)$ may be approximated arbitrarily closely in the $\rho$ metric by elements in $\mathcal{P}^{M}(Q)$ for $M$ sufficiently large.

These ideas and results can then be used to establish a type of "stability" of the inverse problem (see $[5,13])$. We first define a series of approximate problems consisting of minimizing

$$
J\left(P_{M}\right)=\sum_{x=1}^{n}\left|\mathcal{E}\left[x\left(t_{i} ; q\right) \mid P_{M}\right]-\hat{d}_{i}\right|^{2}
$$

over $P_{M} \in \mathcal{P}^{M}(Q)$. Then we have

Theorem 2.3 Let $Q$ be a compact metric space and assume solutions $x(t ; q)$ of $\dot{x}(t)=$ $f(t, x(t), q)$ are continuous in $q$ on $Q$. Let $\mathcal{P}(Q)$ be the set of all probability measures on $Q$ and let $Q_{d}$ be a countable dense subset of $Q$ as defined previously with $Q_{M}=\left\{q_{j}^{M}\right\}_{j=1}^{M}$. Define $\mathcal{P}^{M}(Q)$ as above. Suppose $P_{M}^{*}\left(\hat{d}^{k}\right)$ is the set of minimizers for $J(P)$ over $P \in \mathcal{P}^{M}(Q)$ corresponding to the data $\left\{\hat{d}^{k}\right\}$ and $P^{*}(\hat{d})$ is the set of minimizers over $P \in \mathcal{P}(Q)$ corresponding to $\hat{d}$, where $\hat{d}^{k}, \hat{d} \in R^{n}$ are the observed data such that $\hat{d}^{k} \rightarrow \hat{d}$. Then $\operatorname{dist}\left(P_{M}^{*}\left(\hat{d}^{k}\right), P^{*}(\hat{d})\right) \rightarrow 0$ as $M \rightarrow \infty$ and $\hat{d}^{k} \rightarrow \hat{d}$. Thus the solutions depend continuously on the data and the approximate problems are method stable.

To illustrate the above methodology with a relevant example, we present here a brief description of a PBPK-hybrid model for trichloroethylene (TCE) and indicate how one formulates and implements the corresponding estimation problems. TCE is a metal degreasing agent that is a widespread environmental contaminant, and has been linked to several types of cancer in laboratory animals and humans. This compound is highly soluble in lipids and is known to accumulate within the fat tissue. Therefore, in order to accurately predict toxicity-related measures such as the net clearance rate of TCE and the effective dose of TCE delivered to target tissues, it is important to accurately capture the transport of TCE within the fat tissue.

Physiologically based pharmacokinetic (PBPK) models are used to describe the disposition of compounds such as TCE within the tissues and organs. These models include 
compartments for tissues that are involved in the uptake, metabolism, elimination, and/or transport of the compound, as well as compartments for tissues that are targets of the chemical's toxic effects. See [24] for a detailed description of standard PBPK modeling techniques.

As discussed in [1,27], the standard perfusion-limited and diffusion-limited compartmental models used in PBPK modeling are not able to describe the dynamics of TCE in fat tissue as seen in experimental data, and the assumptions for these ODE-based models do not match well with the heterogeneous physiology of fat tissue. This motivated the development of a specialized compartmental model for the fat tissue, which is then coupled with standard compartments for the remaining non-fat compartments to produce a PBPK-hybrid model.

The resulting compartmental model for the fat tissue is based on an axial dispersion model originally developed by Roberts and Rowland [28] for the transport of solutes in the liver. The underlying assumptions for the dispersion model match well with the physiology of fat tissue (see [1, 27] for details), and the geometry for the PDE-based fat model is based specifically on the known geometry of fat cells and their accompanying capillaries. A key feature of the dispersion model is its aggregate nature, using a representative "cell" to capture the transport behavior of the compound in a collection of many similar "cells" that have varying properties.

In this particular case, the representative "cell" is a unit containing three subcompartments: a single adipocyte (fat cell) together with an adjoining capillary, and the surrounding interstitial fluid. In the model, the adipocyte is represented by a sphere and the capillary is a cylindrical tube with circular cross-section; the interstitial fluid fills in the space surrounding the other two regions. The model geometry and equations are given in spherical coordinates. See $[1,27]$ for a complete description of the model.

It is assumed that TCE enters the capillary region of the fat compartment along with the arterial blood. The capillary equation (2.7) includes a one-dimensional convection-dispersion term together with a term based on Fick's first law of diffusion for the exchange between the capillary and the other two subcompartments. The accompanying boundary conditions (2.8) and (2.9) connect the capillary with the arterial and venous blood systems, and are based on flux balance. The adipocyte and interstitial equations (2.10) and (2.15) each contain two-dimensional diffusion terms together with terms for the exchange of TCE between the subcompartments. The boundary conditions (2.11)-(2.14) and (2.16)-(2.19) are based on standard periodic and finiteness conditions that are appropriate for diffusion on a spherical domain.

In addition to the fat compartment, there are perfusion-limited tissue compartments used in the PBPK-hybrid model to represent the brain, kidney, liver, muscle and remaining tissues. Uptake of TCE is via inhalation in the lungs, which is modeled using a standard steady-state assumption. Metabolism of TCE is described with a Michaelis-Menten term in the liver with parameters $v_{\max }(\mathrm{mg} / \mathrm{hour})$ and $k_{M}(\mathrm{mg} / \mathrm{liter})$. The resulting equations for the PBPK-hybrid model are given by 


$$
\begin{aligned}
& V_{B} \frac{\partial C_{B}}{\partial t}=\frac{V_{B}}{r_{2} \sin \phi} \frac{\partial}{\partial \phi}\left[\sin \phi\left(\frac{\mathcal{D}_{B}}{r_{2}} \frac{\partial C_{B}}{\partial \phi}-v C_{B}\right)\right] \\
& +\lambda_{I} \mu_{B I}\left(f_{I} C_{I}\left(\theta_{0}\right)-f_{B} C_{B}\right) \\
& +\lambda_{A} \mu_{B A}\left(f_{A} C_{A}\left(\theta_{0}\right)-f_{B} C_{B}\right) \\
& -\frac{\mathcal{D}_{B}}{r_{2}} \frac{\partial C_{B}}{\partial \phi}(t, \phi)+\left.v C_{B}(t, \phi)\right|_{\phi=\varepsilon_{1}}=\frac{Q_{c}}{1000 A_{B}} C_{a}(t) \\
& -\frac{\mathcal{D}_{B}}{r_{2}} \frac{\partial C_{B}}{\partial \phi}(t, \phi)+\left.v C_{B}(t, \phi)\right|_{\phi=\pi-\varepsilon_{2}}=\frac{Q_{c}}{1000 A_{B}} C_{v}(t) \\
& V_{I} \frac{\partial C_{I}}{\partial t}=\frac{V_{I} D_{I}}{r_{1}^{2}}\left[\frac{1}{\sin ^{2} \phi} \frac{\partial^{2} C_{I}}{\partial \theta^{2}}+\frac{1}{\sin \phi} \frac{\partial}{\partial \phi}\left(\sin \phi \frac{\partial C_{I}}{\partial \phi}\right)\right] \\
& +\delta_{\theta_{0}}(\theta) \chi_{B}(\phi) \lambda_{I} \mu_{B I}\left(f_{B} C_{B}-f_{I} C_{I}\right)+\mu_{I A}\left(f_{A} C_{A}-f_{I} C_{I}\right) \\
& C_{I}(t, \theta, \phi)=C_{I}(t, \theta+2 \pi, \phi) \\
& \frac{\partial C_{I}}{\partial \theta}(t, \theta, \phi)=\frac{\partial C_{I}}{\partial \theta}(t, \theta+2 \pi, \phi) \\
& C_{I}(t, \theta, 0)<\infty \\
& C_{I}(t, \theta, \pi)<\infty \\
& V_{A} \frac{\partial C_{A}}{\partial t}=\frac{V_{A} D_{A}}{r_{0}^{2}}\left[\frac{1}{\sin ^{2} \phi} \frac{\partial^{2} C_{A}}{\partial \theta^{2}}+\frac{1}{\sin \phi} \frac{\partial}{\partial \phi}\left(\sin \phi \frac{\partial C_{A}}{\partial \phi}\right)\right] \\
& +\delta_{\theta_{0}}(\theta) \chi_{B}(\phi) \lambda_{A} \mu_{B A}\left(f_{B} C_{B}-f_{A} C_{A}\right)+\mu_{I A}\left(f_{I} C_{I}-f_{A} C_{A}\right) \\
& C_{A}(t, \theta, \phi)=C_{A}(t, \theta+2 \pi, \phi) \\
& \frac{\partial C_{A}}{\partial \theta}(t, \theta, \phi)=\frac{\partial C_{A}}{\partial \theta}(t, \theta+2 \pi, \phi) \\
& C_{A}(t, \theta, 0)<\infty \\
& C_{A}(t, \theta, \pi)<\infty \\
& V_{v} \frac{d C_{v}}{d t}=\frac{Q_{m} C_{m}}{P_{m}}+\frac{Q_{t} C_{t}}{P_{t}}+\frac{Q_{f} C_{f}}{P_{f}}+\frac{Q_{b r} C_{b r}}{P_{b r}}+\frac{Q_{l} C_{l}}{P_{l}}+\frac{Q_{k} C_{k}}{P_{k}}-Q_{c} C_{v} \\
& C_{a}=\frac{Q_{c} C_{v}+Q_{p} C_{c}}{Q_{c}+\frac{Q_{p}}{P_{b}}} \\
& V_{m} \frac{d C_{m}}{d t}=Q_{m}\left(C_{a}-C_{m} / P_{m}\right) \\
& V_{t} \frac{d C_{t}}{d t}=Q_{t}\left(C_{a}-C_{t} / P_{t}\right) \\
& V_{b r} \frac{d C_{b r}}{d t}=Q_{b r}\left(C_{a}-C_{b r} / P_{b r}\right)
\end{aligned}
$$




$$
\begin{aligned}
V_{l} \frac{d C_{l}}{d t} & =Q_{l}\left(C_{a}-C_{l} / P_{l}\right)-\frac{v_{\max } C_{l} / P_{l}}{k_{M}+C_{l} / P_{l}} \\
V_{k} \frac{d C_{k}}{d t} & =Q_{k}\left(C_{a}-C_{k} / P_{k}\right) .
\end{aligned}
$$

The variables in the model are the concentrations of TCE (in mg/liter) in each of the compartments/subcompartments, and are denoted by $C$ with subscripts corresponding to the respective tissue/region. Model parameters include tissue volumes $V$ in liters, blood flow rates $Q$ in liters/hour and partition coefficients $P$, each with the appropriate tissue subscripts. Parameters specific to the dispersion model include the dispersion coefficient $\mathcal{D}_{B}$ and the diffusion coefficients $D_{I}$ and $D_{A}$ in $\mathrm{m}^{2} /$ hour; unbound fractions $f_{B}, f_{I}, f_{A}$; permeability coefficients $\mu_{B A}, \mu_{I A}, \mu_{B I}$ in liters/hour; blood flow parameters $v$ (m/hour) and $\mathcal{F}$; and inter-region transport parameters $\lambda_{I}$ and $\lambda_{A}$. A complete discussion of the model equations and parameters is presented in $[1,27]$.

Here we utilize the TCE model (2.7)-(2.26) to illustrate parameter estimation techniques for models with individual-level dynamics that have realization-dependent derivatives. We present results for both parametric and nonparametric parameter estimation approaches, where the parameter of interest is the probability distribution of the fat dispersion coefficient $\mathcal{D}_{B}$ in the capillary. This parameter is an important measure of the degree of heterogeneity within an individual's fat tissue.

The parametric and nonparametric approaches each fit into the general framework presented earlier in this chapter for models with individual dynamics. We assume that the parameter $q \equiv \mathcal{D}_{B} \in Q$ is distributed across the population with distribution $P \in \Pi \subset \mathcal{P}(Q)$, where $\Pi$ is a set of admissible probability distribution functions (possibly all of $\mathcal{P}(Q)$ ). Then the general objective function for the standard least squares parameter estimation problem is given by

$$
J(P)=\sum_{i=1}^{n}\left|\mathcal{E}\left[x\left(t_{i} ; q\right) \mid P\right]-\hat{d}_{i}\right|^{2}
$$

where, in this case, $\hat{d}_{i}$ represents a measurement of the spatial mean concentration of TCE in the fat cells at time $t_{i}$, and $x\left(t_{i} ; q\right)$ is the spatial mean concentration of TCE in the adipocyte region of the fat compartment that is obtained by solving (2.7) - (2.26) with parameter $q$.

For the parametric approach, we assume that the probability distribution $P$ for $q$ is of a particular form with parameterization $\tilde{q} \in R^{N_{q}}$ (e.g., a normal distribution $\mathcal{N}(\mu, \sigma)$ with parameterization $\tilde{q}=(\mu, \sigma))$, so that the set $\Pi$ of admissible probability distributions is defined as the set of all distributions $P_{\tilde{q}}$ of that given form. The estimation problem is then reduced to the $N_{q}$-dimensional problem of minimizing

$$
J(\tilde{q})=\sum_{i=1}^{n}\left|\mathcal{E}\left[x\left(t_{i} ; q\right) \mid P_{\tilde{q}}\right]-\hat{d}_{i}\right|^{2}
$$

over $P_{\tilde{q}} \in \Pi$ for admissible parameterizations $\tilde{q} \in \tilde{Q} \subset R^{N_{q}}$. 
A major advantage of this approach is the reduction of the original infinite-dimensional objective function (2.27) to a more tractable $N_{q}$-dimensional problem. When there is a high degree of confidence about the specific form of the probability distribution $P$, this method can be expected to perform reasonably well. In many cases, however, the exact form of $P$ is unknown, making it difficult to choose the proper restriction for the set $\Pi$ and the corresponding parameterization $\tilde{q}$. If an incorrect form and parameterization are chosen for the distribution function, the parametric approach is likely to provide a poor fit to the data since the "true" underlying distribution may not correspond to a distribution in the admissible set $\Pi$. Even more alarming are situations where a reasonable fit is found even though an incorrect parameteric form has been assumed (see [15] for examples). In this situation, a nonparametric approach is often more appropriate.

Instead of using a specific form for the distribution $P$ with a finite-dimensional parameterization $\tilde{q}$, the nonparametric parameter estimation approach utilizes a discretization of the admissible parameter set $Q$ to achieve a finite-dimensional approximation for the original objective function (2.27). The resulting family of finite-dimensional estimation problems can be solved in a straightforward manner using quadratic programming, and theoretical results established in $[5,15]$ guarantee that the minimizers converge to a minimizer for the infinite-dimensional problem (e.g., see Theorem 2.3 above).

As described earlier in this chapter, we utilize the set $Q_{d}=\bigcup Q_{M}$, a dense, countable subset of $Q$, together with convex combinations of Dirac delta distributions defined over $Q_{M}$, to define the following family of objective functions over $\mathcal{P}^{M}(Q)$ :

$$
J\left(P_{M}\right)=\sum_{i=1}^{N}\left|\mathcal{E}\left[x\left(t_{i} ; q\right) \mid P_{M}\right]-\hat{d}_{i}\right|^{2},
$$

where $\hat{d}_{i}$ are observations corresponding to the expected value, and $P_{M}$ is a probability distribution in $\mathcal{P}^{M}(Q)$ as defined in Theorem 2.3 above.

Note that (2.29) can be rewritten as

$$
J\left(P_{M}\right)=\sum_{i=1}^{N}\left|\sum_{j=1}^{M} x\left(t_{i} ; q_{j}^{M}\right) p_{j}-\hat{d}_{i}\right|^{2},
$$

so that the minimization of (2.30) is equivalent to solving a constrained quadratic programming problem for $\left\{p_{1}, \ldots, p_{M}\right\}$ with constraints $p_{j} \geq 0$ and $\sum_{j=1}^{M} p_{j}=1$.

Example results for the parametric and nonparametric methods are given in Figures 1 and 2 respectively. In each case, the observations used in the parameter estimation problems were generated by solving the TCE model (2.7)-(2.26) with a fixed parameter set $q^{*}$. In this case, the solution $x\left(t_{i} ; q\right)$ is the spatial mean adipocyte concentration of TCE given the parameter $q=\mathcal{D}_{B}$. The probability distributions obtained by the estimation methods are presented in Figures 1 and 2. In Figure 1, the solid line represents the true distribution corresponding to $q^{*}, q_{0}$ denotes the initial iterate used in the optimization procedure and $q$ is the estimated parameterization. 


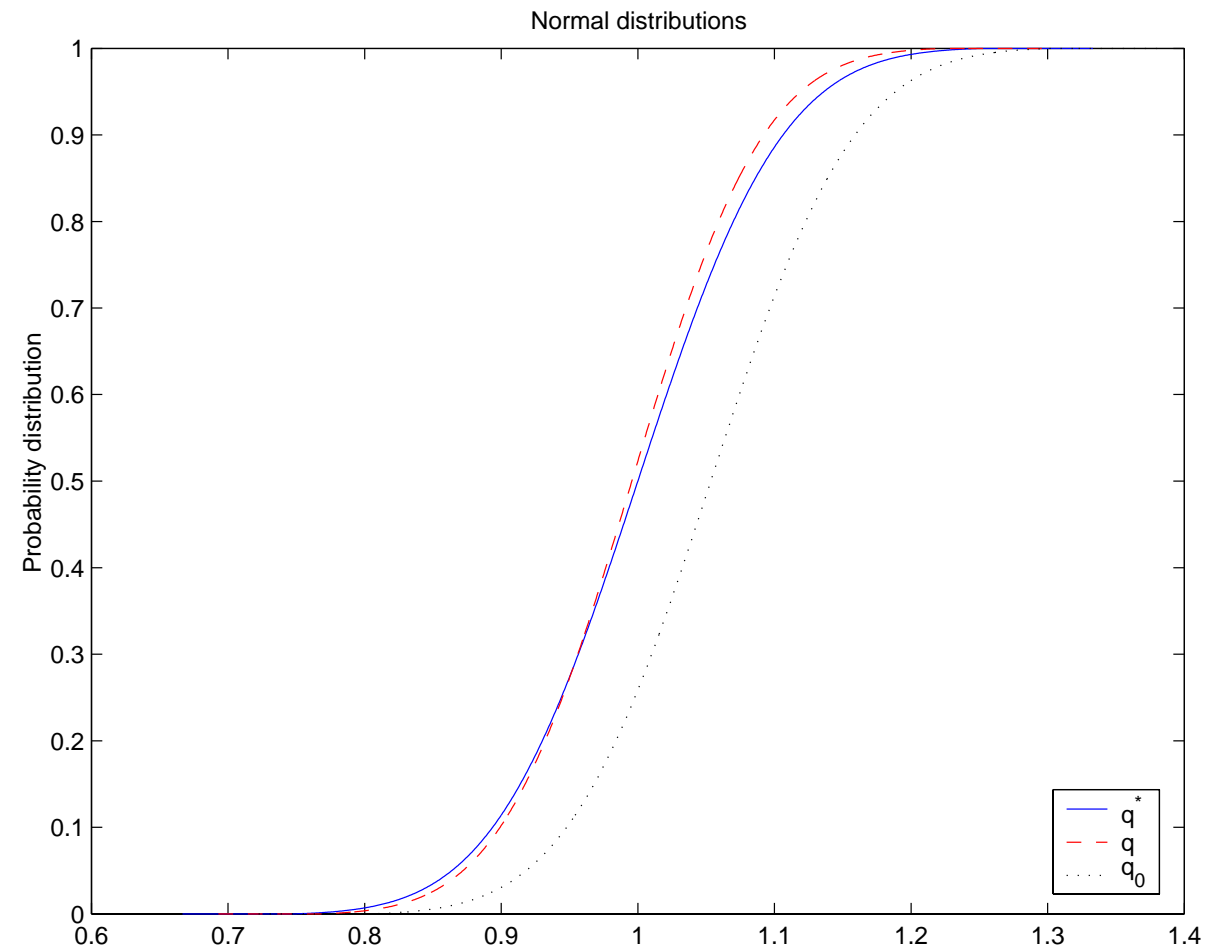

Figure 1: Example solution for the parametric method applied to the TCE PBPK-hybrid model. 


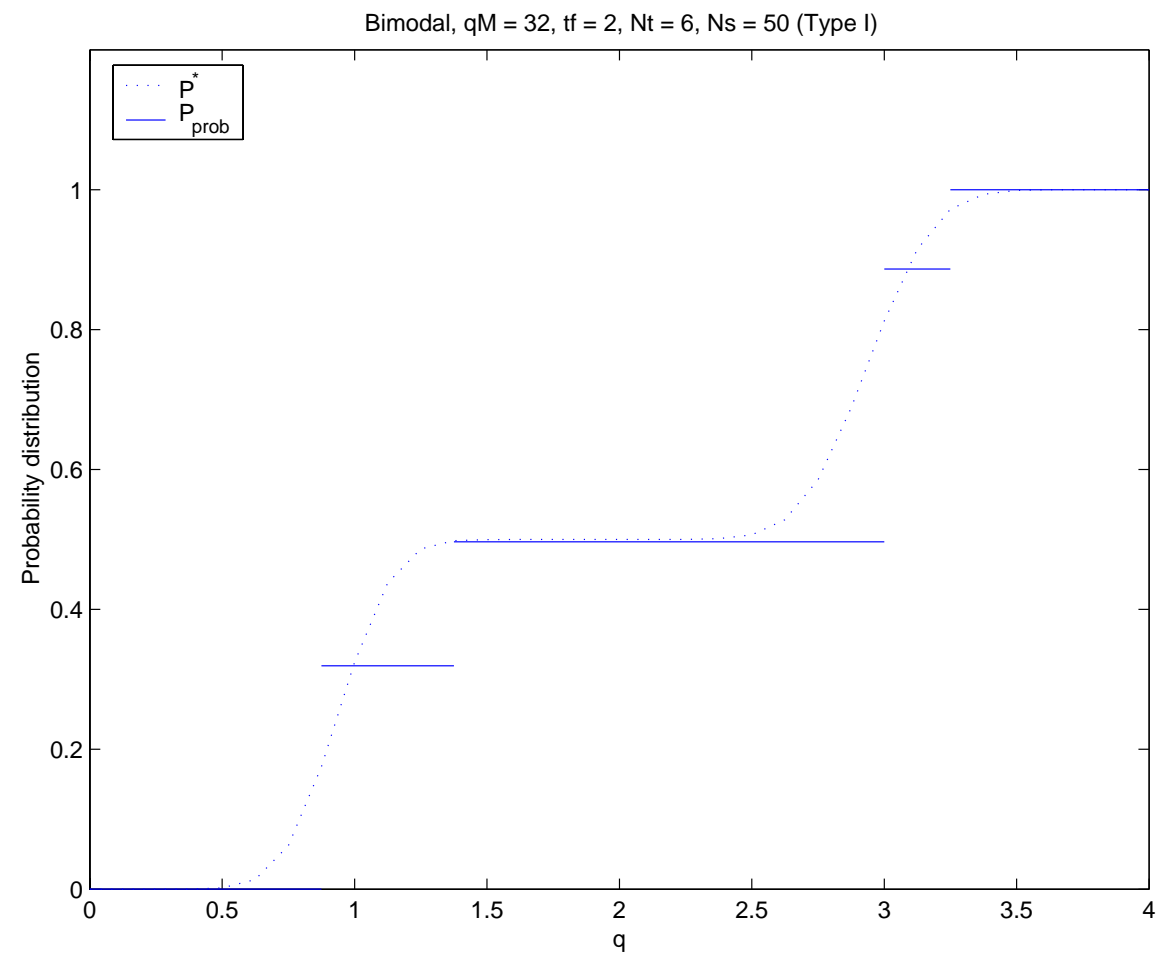

Figure 2: Example solution for the nonparametric method applied to the TCE PBPK-hybrid model.

For the parametric case, the data-generating probability distribution we chose is a bitruncated normal distribution for $q^{*}$ with mean $\mu^{*}=1$, standard deviation $\sigma^{*}=0.0833$, and support over the interval $\left[\mu^{*}-3 \sigma^{*}, \mu+3 \sigma^{*}\right]$. The objective function (2.28) was minimized over the set $\Pi$ of bitruncated normal distributions with parameterizations $(\mu, \sigma)$ and with finite support in $[\mu-3 \sigma, \mu+3 \sigma]$. See [27] for complete details and additional examples.

For the nonparametric case, we used a bimodal gaussian distribution with means $\mu_{1}^{*}=1$ and $\mu_{2}^{*}=3$ and standard deviations $\sigma_{1}^{*}=0.1667$ and $\sigma_{2}^{*}=0.3333$. The objective function (2.30) was minimized using the quadratic programming routine quadprog in Matlab. More details and examples for the nonparametric approach applied to the TCE model are given in [15].

\section{$2.2 \quad$ Aggregate Dynamics}

We turn next to the problems with aggregate dynamics (2.4) and OLS functional (2.5). For these problems one can also develop a general theoretical framework. We first outline the details for ODE systems such as population and SIR models. 


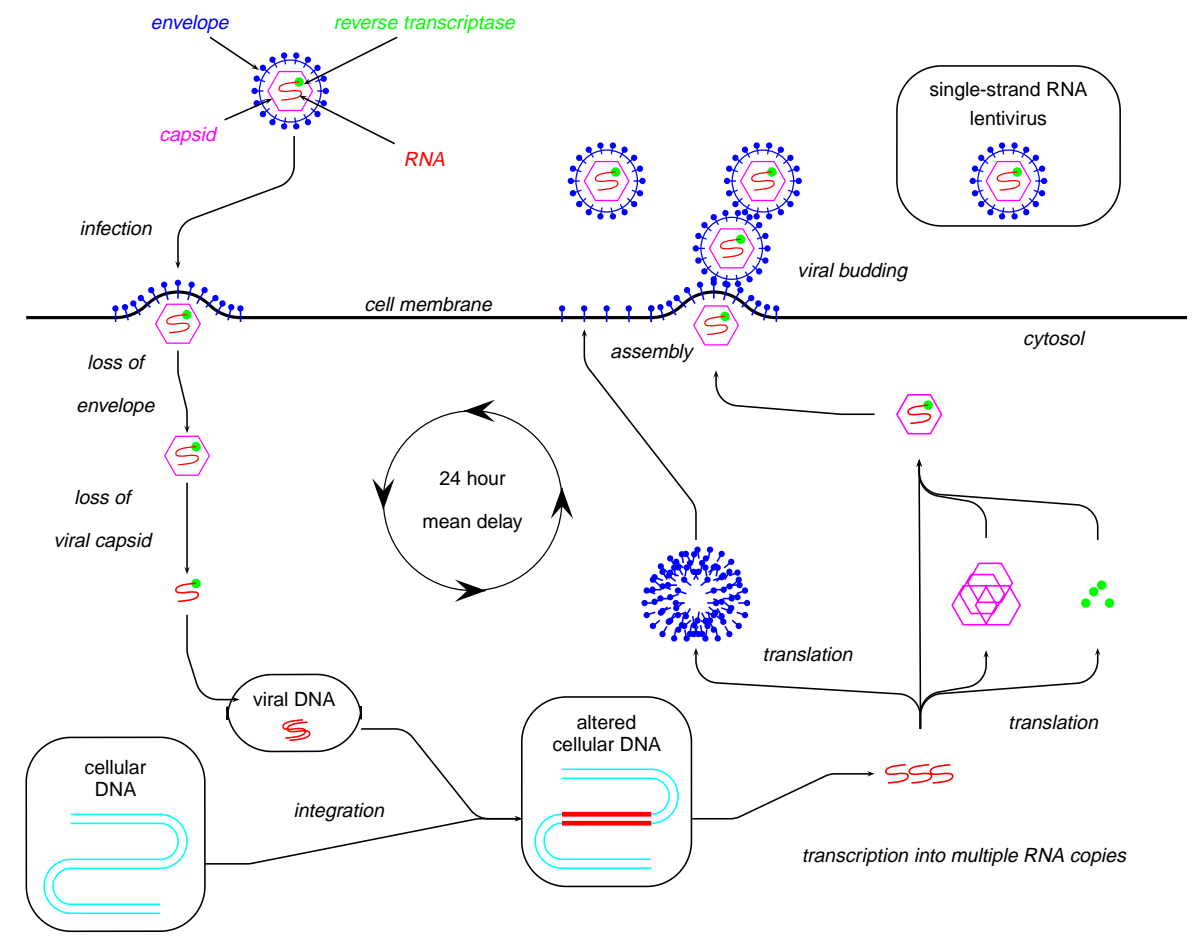

Figure 3: HIV infection pathway.

Given the system dynamics

$$
\frac{d \bar{x}}{d t}=g(t, \bar{x}(t), P), \quad P \in \mathcal{P}(Q),
$$

one first argues that $(t, \bar{x}, P) \rightarrow g(t, \bar{x}, P)$ is continuous from $[0, T] \times R^{n} \times \mathcal{P}(Q)$ to $R^{n}$, and locally Lipschitz in $\bar{x}$. Then by extension of standard continuous dependence on "parameters" results for ODEs, one obtains that $P \rightarrow \bar{x}(t ; P)$ is continuous from $\mathcal{P}(Q)$ to $R^{n}$ for each $t$. This again yields $P \rightarrow J(P)=\sum_{i}\left|\bar{x}\left(t_{i} ; P\right)-\hat{d}_{i}\right|^{2}$ is continuous from $\mathcal{P}(Q)$ to $R^{1}$, where $\mathcal{P}(Q)$, with the Prohorov metric, is compact for $Q$ compact.

Then the general theory of Banks-Bihari [5] can be followed to obtain existence and stability for inverse problems (continuous dependence with respect to data of solutions of the inverse problem) as in Theorems 2.2 and 2.3 above. Moreover, an approximation theory as a basis for computational methods is obtained.

We illustrate the ideas in the situation where the underlying ODE system (2.31) is replaced by a nonlinear functional differential equation (FDE) system. This example arises in modeling progression of HIV for which a schematic of the cellular level infection pathway is given in Figure 3. This is explained more fully in [6, 17]. 
The model is a vector system for the variables $\bar{x}(t)=(V(t), A(t), C(t), T(t))$ given by

$$
\begin{aligned}
& \dot{V}(t)=-c V(t)+n_{A} \int_{-r}^{0} A(t+\tau) d P_{1}(\tau)+n_{C} C(t)-p V(t) T(t) \\
& \dot{A}(t)=\left(r_{v}-\delta_{A}-\delta X(t)\right) A(t)-\gamma \int_{-r}^{0} A(t+\tau) d P_{2}(\tau)+p V(t) T(t) \\
& \dot{C}(t)=\left(r_{v}-\delta_{C}-\delta X(t)\right) C(t)+\gamma \int_{-r}^{0} A(t+\tau) d P_{2}(\tau) \\
& \dot{T}(t)=\left(r_{u}-\delta_{u}-\delta X(t)-p V(t)\right)(t)+S(t),
\end{aligned}
$$

where $X=A+C+T$ and $V(t)$ is the expected value of the population count (number) of virus cells, $A(t)$ is the number of acutely infected cells, $C(t)$ is the expected value of the number of chronically infected cells, and $T(t)$ is the total number of target or uninfected cells, each at time $t$ respectively. The probability measures $P_{1}, P_{2}$ in the model arise because there are delays $\tau_{1}$ and $\tau_{1}+\tau_{2}$ from the time of acute cellular infection until a cell becomes productively infected and from the time of acute infection until chronic infection, respectively (see Appendix A of [6] or Chapter 2 of [17] for a careful and detailed derivation). Biologically, these delay times must vary across the population and this variability is described by the PDFs $P_{1}$ and $P_{2}$ in the system (2.32)- (2.35). More specifically, the variables $V(t)$ and $C(t)$ have substructures (classes $V(t ; \tau), C(t ; \tau)$ grouped according to their or their "mothers" delay times) which are averaged across the populations using the distributions $P_{1}, P_{2}$, respectively, so that

$$
\begin{aligned}
& V(t)=\mathcal{E}\left[V(t ; \tau) \mid P_{1}\right]=\int_{-r}^{0} V(t ; \tau) d P_{1}(\tau), \\
& C(t)=\mathcal{E}\left[C(t ; \tau) \mid P_{2}\right]=\int_{-r}^{0} C(t ; \tau) d P_{2}(\tau) .
\end{aligned}
$$

This yields the system (2.32)-(2.35) with vector valued measure dependent $\left(P=\left(P_{1}, P_{2}\right)\right)$ dynamics as formulated in (2.31) wherein the "state" variables are expected values of population counts. A careful consideration of the derivation of this system reveals that it does not arise from a parameter dependent system for

$$
x(t ; q)=(V(t ; q), A(t ; q), C(t ; q), T(t ; q))
$$

with parameters $q=\left(\tau_{1}, \tau_{2}\right)$ and thus the associated inverse problems for the estimation of $P_{1}, P_{2}$ are fundamentally different from those in the PBPK examples of Section 2.1 above.

The dynamical system (2.32)-(2.35) for given $P_{1}, P_{2}$ is itself an infinite-dimensional state system (similar to a partial differential equation (PDE) system in this regard). To see this, we note that $(2.32)-(2.35)$ can be written (see $[6,17]$ for details) in the form

$$
\dot{\bar{x}}(t)=L\left(\bar{x}(t), \bar{x}_{t}\right)+f_{1}(\bar{x}(t))+f_{2}(t), \quad t \geq 0,
$$




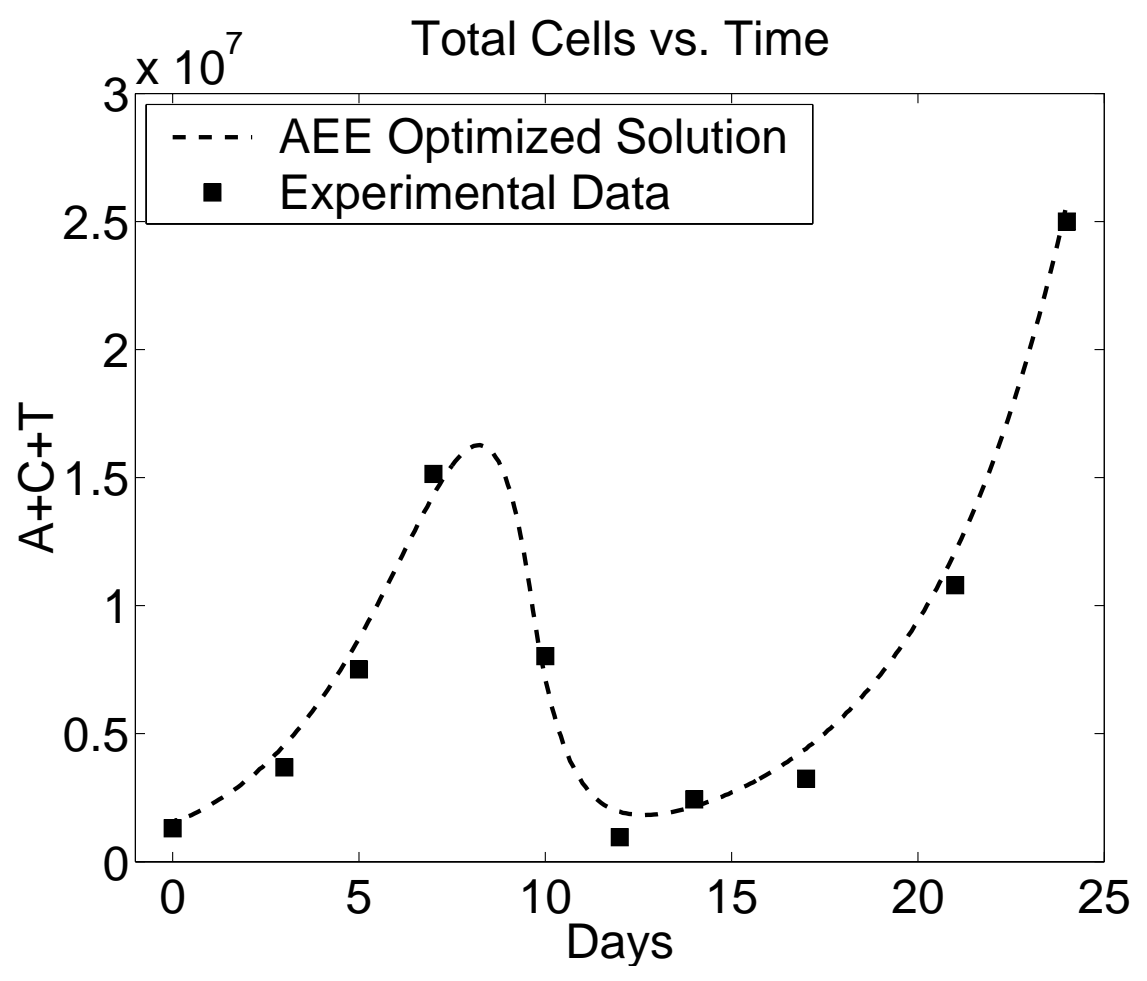

Figure 4: Data versus best fit simulation. 
where $\theta \rightarrow \bar{x}_{t}(\theta) \equiv \bar{x}(t+0),-r \leq \theta \leq 0$, is a function from $[-r, 0]$ to $R^{4}$. This system requires initial data $\left(\bar{x}(0), \bar{x}_{0}\right)$ in the state space $\tilde{Z}=R^{4} \times \mathcal{C}\left(-r, 0 ; R^{4}\right)$ which is readily recognized as being infinite dimensional. For such systems one needs an approximation theory and resulting computational methodology (e.g., finite element methods similar to those popular in PDE theory and implementation) even to carry out forward simulations (an integral part, of course, of most inverse problem methodologies). Fortunately, such a theory exists [4, 9, 10] in the context of abstract evolution equations

$$
\dot{z}(t)=\mathcal{A} z(t)+\left(f_{2}(t), 0\right)
$$

in a state space $Z=R^{4} \times L_{2}\left(-r, 0 ; R^{4}\right)$ where

$$
\mathcal{D}(\mathcal{A})=\left\{(\phi(0), \phi) \in Z: \phi \in H^{1}\left(-r, 0 ; R^{4}\right)\right\}
$$

and $\mathcal{A}: \mathcal{D}(\mathcal{A}) \subset Z \rightarrow Z$ is given by

$$
\mathcal{A}(\phi(0), \phi)=\left(L(\phi(0), \phi)+f_{1}(\phi(0)), \frac{d}{d \theta} \phi\right)
$$

for $\theta \rightarrow \phi(\theta)$ in $H^{1}\left(-r, 0 ; R^{4}\right)$.

This theory can be used as a foundation to develop a theoretical and computational framework for inverse problems similar to that outlined for parameter dependent systems such as the PBPK example in Section 2.1. While the resulting wellposedness and method stability (see Chapter 3 of [17]) statements are similar in spirit to the Banks-Bihari results given in Section 2.1, the technical details are quite different and rely heavily on the FDE theory in $[4,9,10]$. Details are given in $[6,17]$.

The methodology outlined here (along with an ANOVA type statistical methodology) was successfully used to analyze in vitro data [29] from the experiments of Dr. Michael Emerman of the Fred Hutchinson Cancer Research Center in Seattle. A comparison of the simulation of the model with minimizing $P^{*}=\left(P_{1}^{*}, P_{2}^{*}\right)$ obtained from the inverse problem (i.e., (2.5) with system (2.32)-(2.35)) to a set of Emerman's experimental data is depicted in Figure 4 . We note that the measures $P_{1}^{*}, P_{2}^{*}$ used for the simulation depicted here consisted of Dirac measures with single atoms at $\tau_{1}^{*}$ and $\tau_{1}^{*}+\tau_{2}^{*}$, respectively, where $\tau_{1}^{*}=22.8$ hours and $\tau_{2}^{*}=3.2$ hours.

\section{$3 \quad$ Electromagnetic imaging of hidden substances}

In this section we summarize our efforts in modeling the use of electromagnetic pulsed signals to remotely extract information about geometric and chemical properties of substances. Our goal is to describe some existing methodologies developed for the propagation of microwaves 
in dielectric materials and related imaging techniques as well as to outline some directions in which this theory is currently being extended.

The interaction of very high frequency electromagnetic waves, X-rays, with materials has long been exploited for imaging purposes in medical diagnostics. Many novel techniques have been developed during the past several years to extend the capabilities of traditional X-ray methods. Moreover, waves at different frequency ranges of the electromagnetic spectrum have been utilized. A close inspection of the interaction of materials with electromagnetic radiation at different frequency ranges reveals different underlying mechanisms which need to be correctly captured in the appropriate models. At the same time, the diversity of this interaction makes possible a variety of applications from laser surgery to the detection of environmental contaminants. Some of these techniques have great potential to play an important role in the current efforts in providing a more secure environment from different forms of terrorist activities. As stated in Section 1, interrogation of materials with electromagnetic waves could be useful in look-down surveillance, imaging of structures, identification of contaminants, airport security devices, detection of hidden substances, explosives, chemicals, toxins and bioagents.

The successful use of these techniques is wrought with many technical and theoretical challenges. While portable lasers and X-ray machines are widely available, other ranges of the EM spectrum are not as well represented. Terahertz signal generators and detectors are currently being developed and exhibit a great promise for providing novel imaging devices. Terahertz radiation has several advantages over traditional X-ray methods and is well-suited for imaging applications. T-rays have low photon energies and are non-ionizing, thus they are thought to be safer than X-rays. Recently developed devices can generate very short (sub-ps) bursts of $\mathrm{THz}$ radiation consisting of only a few cycles of the electric field, yet spanning a broad bandwidth. THz waveforms passing through, or reflected from an object can be recorded in the time domain with very high signal-to-noise ratio. Many organic molecules show strong absorption and dispersion in this frequency range. These effects constitute the polarization mechanism of the molecules which has an influence on the electric field and the propagation of the electromagnetic wave inside the material. Since these transitions are characteristic to the particular molecules, detection of the temporal distortions produced thus yields information about the composition of the material in real time. For example, it is known that cancerous and benign tumors have different electromagnetic characteristics. Therefore an imaging device based on $\mathrm{THz}$ waves could not only give information about the structure of an object (geometrical properties) but could help in determining their composition and electromagnetic properties as well in a non-invasive way. As shown in [25], T-ray imaging can be useful both by sending a pulse through the material and detecting it on the other side or by sending a pulse toward the material and recording the reflections from the interface(s) (reflection imaging). This latter procedure is especially important when detectors cannot be placed on the other side of the object, or when only slices of an object need to be evaluated. Potential applications range from medical and dental diagnostics to quality control in food processing, semiconductor and chip manufacturing and to the detection of hidden objects and substances in containers. It has been demonstrated in [32] that terahertz 
imaging can potentially be used to identify specific powders in mail.

The technical advances in generating electromagnetic radiation in different ranges of the spectrum and their emerging applications in both medical and general imaging fields call for better theoretical understanding and accurate models of the interaction of electromagnetic signals and various substances. In developing these models special attention has to be placed on the specific frequency range and intensity of the electromagnetic radiation, the type of the interrogating signal that is used and the type of material that it encounters. For example, in the high optical range one generally assumes a nonlinear relationship between the electric field and the polarization, and uses the slowly-moving envelope assumption to derive the nonlinear Schrödinger equation for the propagation of wave-packets in a dielectric medium from Maxwell's equations. While the latter is a reasonable assumption for pulses that are "long" compared to a characteristic frequency, it may be inadequate to account for ultrashort pulses. In that case a different, full-wave derivation is necessary to capture transient effects.

In the microwave range of the electromagnetic spectrum one can assume that the relationship between the electric field and the polarization is linear for most materials. In the following we will summarize a model developed in [8] for the propagation of windowed microwave (3-100 GHz) pulses in a dielectric medium. In that work the basic question, which was answered in the affirmative, was whether a variational formulation of Maxwell's equations for a specific 1-D situation could successfully be used in the identification of geometric and dielectric properties of a material slab that is interrogated by microwave pulses from antenna sources.

\subsection{Variational approach for microwave pulse propagation}

In this $1-\mathrm{D}$ model an infinite slab of material is placed in the interval $\Omega=\left[z_{1}, z_{2}\right]$ with faces parallel to the $x y$ plane. The interrogating signal is assumed to be a short planar electromagnetic pulse normally incident on the material and the electric field is polarized with oscillations in the $x z$ plane only.

Thus the electric field is parallel to the $\hat{\imath}$ axis at all points in $\Omega_{0}$ and the magnetic field $\vec{H}$ is parallel to the $\hat{\jmath}$ axis. Since the material properties are assumed to be homogeneous in the $x$ and $y$ variables, it can be shown that the propagating waves in $\Omega$ are also reduced to one nontrivial component [8]. This makes it possible to represent the fields in $\Omega$ and $\Omega_{0}$ with the scalar functions $E(t, z)$ and $H(t, z)$. Under these assumptions, Maxwell's equations reduce to

$$
\begin{aligned}
\frac{\partial E}{\partial z} & =-\mu_{o} \frac{\partial H}{\partial t} \\
-\frac{\partial H}{\partial z} & =\frac{\partial D}{\partial t}+\sigma E+J_{s}
\end{aligned}
$$

for the scalar fields $E$ and $H$. The magnetic field can be eliminated from the equations by

taking the spatial derivative of equation (3.37), the time derivative of equation (3.38) and 


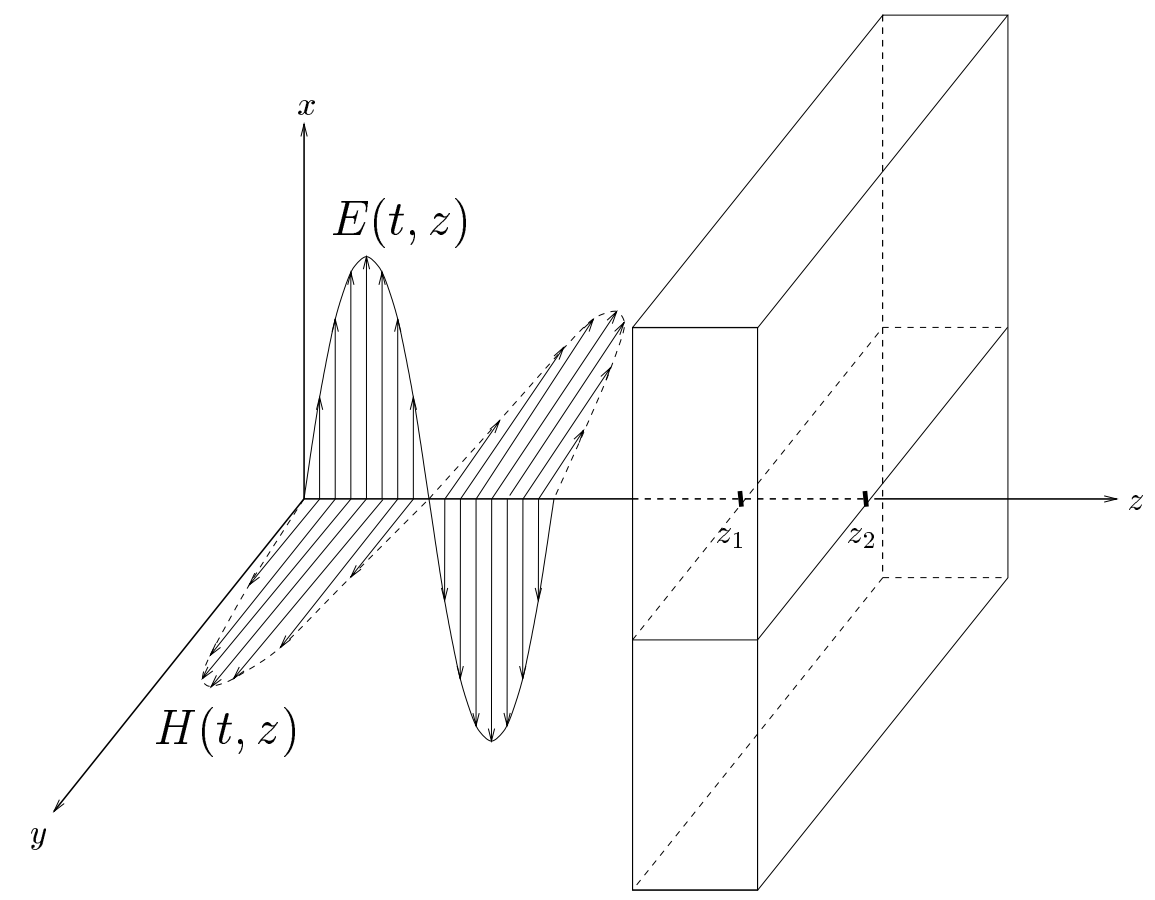

Figure 5: Geometry of the physical problem.

using the equation for electric flux density $D=\epsilon E+P$ where $\epsilon=\epsilon_{0}\left(1+\left(\epsilon_{r}-1\right) I_{\Omega}\right)$ to obtain

$$
\mu_{0} \epsilon \ddot{E}+\mu_{0} \ddot{P}+\mu_{0} \sigma \dot{E}-E^{\prime \prime}=-\mu_{0} \dot{J}_{s} .
$$

A general integral equation model can be employed to describe the behavior of the media's macroscopic electric polarization $P$ :

$$
P(t, x)=\int_{0}^{t} g(t-s, x) E(s, x) d s .
$$

This constitutive law is given in terms of a susceptibility kernel $g$, and expresses the fact that the material responds to the electric field in finite time. This formulation is sufficiently general to include microscopic polarization mechanisms such as dipole or orientational polarization as well as ionic and electronic polarization (see later) $[3,21]$. We note that $P(0, x)$ is assumed to be 0 . To allow for a component of the polarization which depends instantaneously on the electric field one can include a term $\epsilon_{0} \chi E$ in $D$. Hence,

$$
\begin{aligned}
D & =\epsilon_{0}(1+\chi) E+P \\
& =\epsilon_{0} \epsilon_{r} E+P,
\end{aligned}
$$

where $\epsilon_{r}=1+\chi \geq 1$ is a relative permittivity which can be treated as a spatially dependent parameter. 
In this problem the location of the boundary at $z=z_{1}$ is assumed known, while the location of the original back boundary at $z=z_{2}$, i.e., the depth of the slab, is unknown. The unknown boundary creates computational difficulties in the inverse problem since changing domains would involve changing discretization grids in the usual finite element schemes. Thus the method of mappings $[11,12,26]$ is applied to transform the problem to a known reference domain. The domain of the computation is defined to be the interval $\tilde{\Omega}=[0,1]$. An absorbing boundary condition is placed at the $z=0$ boundary of the interval to prevent the reflection of waves. This can be expressed by

$$
\left[\frac{1}{c} \frac{\partial E}{\partial t}-\frac{\partial E}{\partial z}\right]_{z=0}=0
$$

where $c^{2} \equiv 1 / \epsilon_{0} \mu_{0}$. A supraconductive backing is placed on the slab at $z=z_{2}$. The boundary conditions on this supraconductive reflector (after mapping $z_{2}$ to the reference point $z=1$ ) are given by $E(t, 1)=0$. Substituting an expression for $\ddot{P}$ derived from equation (3.40) we obtain the strong form of the equation

$$
\begin{aligned}
& \tilde{\epsilon}_{r} \ddot{E}(t, z)+\frac{1}{\epsilon_{0}} I_{\Omega}(z)(\sigma(z)+g(0, z)) \dot{E}(t, z) \\
& +\frac{1}{\epsilon_{0}} I_{\Omega}(z) \dot{g}(0, z) E(t, z)+\int_{0}^{t} I_{\Omega}(z) \frac{1}{\epsilon_{0}} \ddot{g}(t-s, z) E(s, z) d s \\
& -c^{2} E^{\prime \prime}(t, z)=-\frac{1}{\epsilon_{0}} \dot{J}_{s}(t, z),
\end{aligned}
$$

where indicator functions $I_{\Omega}$ have been added to explicitly enforce the restriction of polarization and conductivity to the interior of the transformed medium $\Omega=\left[z_{1}, 1\right]$ and $\tilde{\epsilon}_{r}=\epsilon / \epsilon_{0}=1+\left(\epsilon_{r}-1\right) I_{\Omega} \geq 1$ throughout $[0,1]$.

Due to the form of the interrogating inputs, the dielectrically discontinuous medium interfaces, and the possible lack of smoothness in mapping the original domain $\Omega_{0} \bigcup \Omega=\left[0, z_{2}\right]$ to the reference domain $\tilde{\Omega}=[0,1]$, one should not expect classical solutions to Maxwell's equations in strong form. Thus it is desirable to write the system equations in weak or variational form. Using the spaces $H=L_{2}(0,1)$ and $V=H_{R}^{1}(0,1)=\left\{\phi \in H^{1}(0,1) \mid \phi(1)=0\right\}$ and the boundary conditions (3.43), the equation (3.44) can be written in weak form as

$$
\begin{aligned}
& \left\langle\tilde{\epsilon}_{r} \ddot{E}, \phi\right\rangle+\langle\gamma \dot{E}, \phi\rangle+\langle\beta E, \phi\rangle+\left\langle\int_{0}^{t} \alpha(t-s, \cdot) E(s, \cdot) d s, \phi\right\rangle \\
& \quad+\left\langle c^{2} E^{\prime}, \phi^{\prime}\right\rangle+c \dot{E}(t, 0) \phi(0)=\langle\mathcal{J}(t, \cdot), \phi\rangle
\end{aligned}
$$

with initial conditions

$$
E(0, z)=\Phi(z) \quad \dot{E}(0, z)=\Psi(z)
$$

where the coefficients are given by

$$
\begin{aligned}
& \alpha(t, z)=\frac{1}{\epsilon_{0}} I_{\Omega}(z) \ddot{g}(t, z), \quad \beta(z)=\frac{1}{\epsilon_{0}} I_{\Omega}(z) \dot{g}(0, z), \\
& \gamma(z)=\frac{1}{\epsilon_{0}} I_{\Omega}(z)(\sigma(z)+g(0, z)), \quad \mathcal{J}(t, z)=-\frac{1}{\epsilon_{0}} \dot{J}_{s}(t, z),
\end{aligned}
$$


and $\langle\cdot, \cdot\rangle$ is the $L^{2}$ inner product. The functions $\alpha, \beta$ and $\gamma$ are dependent on parameters which must be identified. These functions are assumed to be in $L^{\infty}$ but may lack any additional regularity.

Existence, uniqueness and regularity of solutions is established in [8], and a comprehensive approximation framework is developed for the forward as well as the inverse problems. It is shown computationally that it is possible to simulate and identify Debye and Lorentz polarization mechanisms in media using first reflected pulses. The thickness of a layered slab using reflected signals from a supraconductive back boundary can also be accurately estimated. It is demonstrated computationally that this model captures transient effects and shows the formation of Brillouin precursors inside the material [8].
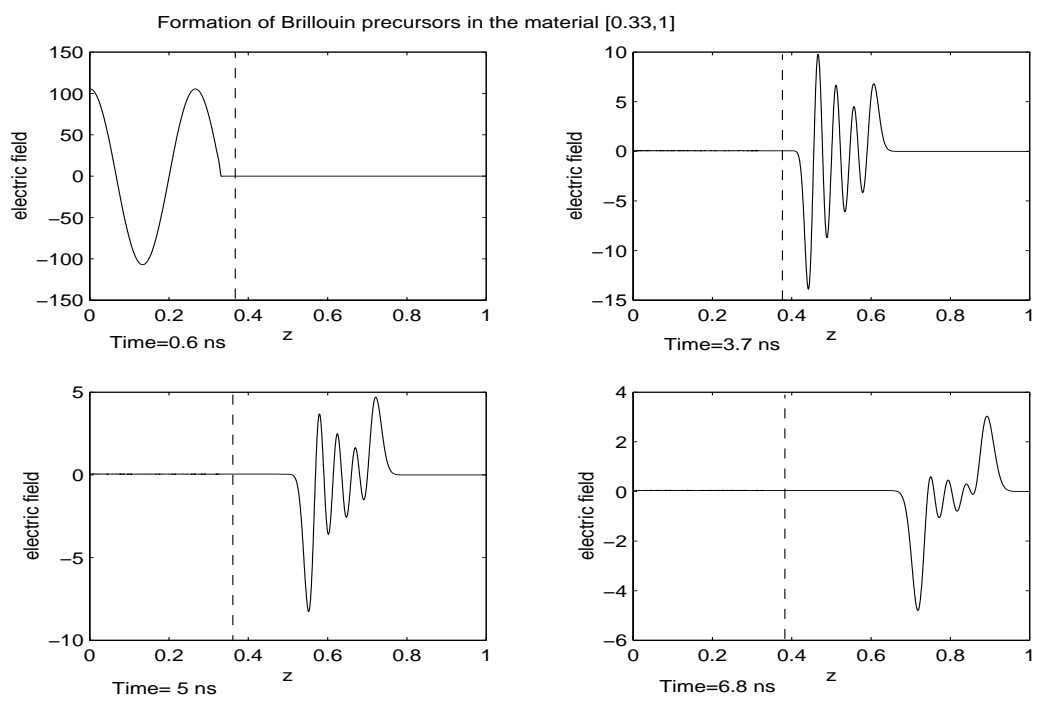

Figure 6: Formation of Brillouin precursors using a linear Debye model.

In summary, this approach is amenable to ultrashort input pulses and provides a complete theoretical and computational framework for the direct and the inverse problem in this onedimensional model.

This work has been extended in different directions. A corresponding analysis with acoustic reflectors at the back of the slab of material and pressure dependent Maxwell system coefficients is developed in [2]. It is shown that instead of a supraconductive backing (which is not practical in many medical or remote imaging applications), an acoustic wave can be employed to reflect the electromagnetic signal. Moreover, these reflections can again be used to identify geometric and dielectric properties of the material.

To develop and use a similar methodology for terahertz signals we must capture the response of materials to higher frequencies. Thus, we need to represent the absorption and dispersion properties of the material by accurately modeling the underlying polarization mechanisms. As interrogating frequencies increase, it is not unreasonable to expect that nonlinearities play a nontrivial role. 


\subsection{Polarization mechanisms}

Polarization, the general macroscopic response of a material to an electric field, is an important dielectric characteristic specific to a given material and hence is important to any interrogation methodology. It depends heavily on the molecular structure of the material.

Dielectric materials contain bound negative and positive charges that are not free to move as charges do in conductors. These charges are kept in place by atomic and molecular forces. When subjected to an external electric field, dipole moments are induced in the atoms and molecules. The electric polarization vector is defined as the dipole moment per unit volume. The mechanism by which these dipole moments are created is different in different materials, whether gases, liquids, or solids. Molecules of certain gases (e.g., oxygen) contain a symmetric pair of atoms in each molecule and thus have no inherent dipole moments. Such molecules are called nonpolar. In others, (e.g., water vapor) the center of gravity of the positive charge (in this case on the hydrogen atom) and the negative charge (on the oxygen) do not coincide, and the total charge distribution on the molecule has a dipole moment. These molecules are called polar.

First we consider nonpolar molecules. When an electric field is applied to the atoms of such molecules the electrons are forced in one direction, while the nucleus is forced in the opposite direction by the field. Thus there is a net displacement of the centers of charge, and a dipole moment is created. This displacement of the electron distribution is called electronic polarization. In a changing electric field the displacement of the center of charge of the electrons is usually modeled by a harmonic oscillator and this gives rise to the Lorentz model for electronic polarization:

$$
\ddot{\vec{P}}+\frac{1}{\tau} \dot{\vec{P}}+\omega_{0}^{2} \vec{P}=\epsilon_{0} \omega_{p}^{2} \vec{E}
$$

where $\epsilon_{0}$ is the dielectric constant, and $\omega_{p}$ is the so called plasma frequency given by $\omega_{p}=$ $\sqrt{\epsilon_{s}-\epsilon_{\infty}}$, with $\epsilon_{s}$ and $\epsilon_{\infty}$ being the relative permittivities of the material in the limit of the static and very high frequencies, respectively.

The same mechanism can be observed in polar molecules. However, in addition to this effect, the electric field forces a portion of the originally randomly oriented internal dipoles to line up with the applied field, producing a net moment per unit volume. This is called dipole or orientational polarization, and is described by the Debye model which captures the relaxation of the molecules once the electric field is turned off:

$$
\tau \dot{\vec{P}}+\vec{P}=\epsilon_{0}\left(\epsilon_{s}-\epsilon_{\infty}\right) \vec{E}
$$

It takes time for the molecules to line up because of their moment of inertia, so this mechanism becomes less pronounced if the material is subjected to very high frequencies. In that case the molecules simply cannot follow the changing electric field sufficiently fast and at some level appear to "freeze."

Polarization in denser materials, liquids and solids, is even more complicated. Here the electric field around each molecule is influenced not only by the external field but the field 
of the neighboring molecules as well, giving rise to vibrational polarization (a form of electronic polarization). In solids that are made up of ionic crystals, e.g., $\mathrm{NaCl}$, the positive and negative ions are displaced as a result of an applied field, which is called ionic polarization. In certain crystals there is a permanent internal polarization in the sense that each unit cell of the lattice has a permanent dipole moment. If the relative position of the lattice points change, e.g., by heating or stressing the material, external fields appear creating pyroelectricity and piezoelectricity, respectively. For an ideal dielectric, orientational polarization dominates for lower frequencies giving way to vibrational and electronic polarization as the frequency increases. At very high frequencies (X-rays, gamma rays) there is almost no polarization since the material simply cannot "follow the wave" due to inertial effects.

In all of these models so far we assumed that the relationship between the applied electric field and the polarization is linear, given by, in general, an integral convolution. However, it is known that in the optical range this relationship becomes nonlinear (more so for non infinitesimal fields), as evidenced by nonlinear optical effects like solitons, second harmonic generation and self-focusing [31]. For some materials this transition starts to take place in the IR range. For example, while for microwaves a linear model is appropriate (indeed a Debye model provides a good fit for water), nonlinear effects, especially for non-infinitesimal amplitudes, need to be taken into account for higher frequency ranges. There is experimental evidence for small but significant departure from strict linearity at high values of the electric field [30] (p. 245). An example is the Kerr effect, in which insulating liquids, containing anisotropic molecules, become doubly refracting when subjected to very strong fields. As suggested in [30], this could be modeled by the constitutive relation $\vec{P}=\vec{E}+s|\vec{E}|^{2} \vec{E}$. However, we have already seen that inertial effects, i.e., the finite time response of the material may be important, so instead we will consider a Debye model where the electric field provides nonlinear forcing. For a centrosymmetric medium we might assume

$$
\tau \dot{\vec{P}}+\vec{P}=\tilde{f}(\vec{E})
$$

where $\tilde{f}(\vec{E})=c_{1} \vec{E}+c_{2}|\vec{E}|^{2} \vec{E}$, for $|\vec{E}|<M$ and 0 otherwise, i.e., $\tilde{f}$ is a saturated cubic nonlinearity. In integral form we obtain the relationship

$$
\vec{P}(t, \vec{x})=\int_{0}^{t} g(t-s, \vec{x}) \tilde{f}(\vec{E}(s, \vec{x})) d s,
$$

where $g(t, \vec{x})=e^{\frac{-t}{\tau}}$. We note that a nonlinearly driven Lorentz model,

$$
\ddot{\vec{P}}+\frac{1}{\tau} \dot{\vec{P}}+\omega_{0}^{2} \vec{P}=\epsilon_{0} \omega_{p}^{2} \tilde{f}(\vec{E})
$$

leads to a similar integral representation with kernel function $g(t, \vec{x})=\frac{\epsilon_{0} \omega_{p}^{2}}{\nu_{0}} e^{-\frac{1}{2 \tau} t} \sin \left(\nu_{0} t\right)$, where $\nu_{0}=\sqrt{\omega_{0}^{2}-\frac{1}{4 \tau^{2}}}$. As a first step, we considered a general model with nonlinear polarization in [14]. 


\subsection{Variational formulation of the model with nonlinear polariza- tion}

We consider a polarization mechanism of the form (3.47) with $\tilde{f}=E+f(E)$ together with the one-dimensional model outlined above. As before, an infinite slab of material with supraconductive backing is interrogated by a normally incident polarized plane wave windowed pulse originating at an antenna source $z=0$ in free space $\Omega_{0}=\left[0, z_{1}\right]$. The slab of material in $\Omega=\left[z_{1}, z_{2}\right]$ is assumed to be homogeneous in the directions orthogonal to the direction $z$ of propagation of the plane wave. As we have already noted, under these assumptions it is possible to represent the strength of the electric and magnetic fields in $\Omega$ and $\Omega_{0}$ by the scalar functions $E(t, z)$ and $H(t, z)$, respectively. One can readily eliminate the magnetic field from the full Maxwell equations and substitute the assumed constitutive law for the polarization to arrive at the strong formulation of the problem with similar initial and boundary conditions as in Section 3.1:

$$
\begin{aligned}
\hat{\varepsilon}_{r} \ddot{E}(t, z)+ & \frac{1}{\varepsilon_{0}} I_{\Omega}(z)(\sigma(z)+g(0, z)) \dot{E}(t, z) \\
& +\frac{1}{\varepsilon_{0}} I_{\Omega}(z) \dot{g}(0, z) E(t, z)+\int_{0}^{t} \frac{1}{\varepsilon_{0}} I_{\Omega}(z) \ddot{g}(t-s, z) E(s, z) d s \\
& +\frac{1}{\varepsilon_{0}} I_{\Omega}(z) \dot{g}(0, z) f(E(t, z))+\int_{0}^{t} \frac{1}{\varepsilon_{0}} I_{\Omega}(z) \ddot{g}(t-s, z) f(E(s, z)) d s \\
& +\frac{1}{\varepsilon_{0}} I_{\Omega}(z) g(0, z) \frac{d}{d t} f(E(t, z))-c^{2} E^{\prime \prime}(t, z) \\
& =-\frac{1}{\varepsilon_{0}} \dot{J}_{s}(t, z), \quad t>0, \quad 0<z<z_{2}, \\
& {\left[\frac{1}{c} \frac{\partial E}{\partial t}-\frac{\partial E}{\partial z}\right] \quad=0 \quad t>0, } \\
& E\left(t, z_{2}\right)=0 \quad t>0, \\
& E(0, z)=\Phi(z), \quad \dot{E}(0, z)=\Psi(z) \quad 0<z<z_{2} .
\end{aligned}
$$

In the physical problem $z_{2}$ is assumed to be unknown, and it is desirable to estimate it from given data. Since the theoretical analysis is constructive in the sense that the numerical method we use to solve this problem (for both forward and inverse problems) follows the theoretical arguments, it is desirable to convert the problem to a fixed spatial domain, e.g., $[0,1]$, as explained above and in [8]. Thus we use the method of maps and subsequently formulate the variational problem as follows.

We let $H=L^{2}(0,1), \quad V=H_{R}^{1}(0,1)=\left\{\phi \in H^{1}(0,1) \mid \phi(1)=0\right\}$ leading to the Gelfand triple $([23,33]) V \hookrightarrow H \hookrightarrow V^{*}$. We say that $E \in L^{\infty}(0, T ; V)$ with $\dot{E} \in L^{2}(0, T ; H), \ddot{E} \in$ $L^{2}\left(0, T ; V^{*}\right)$, is a weak solution if it satisfies for every $\varphi \in V$

$$
\left\langle\overline{\varepsilon_{r}} \ddot{E}, \varphi\right\rangle_{V^{*}, V}+\langle\gamma \dot{E}, \varphi\rangle+\langle\beta E, \varphi\rangle+\left\langle\int_{0}^{t} \alpha(t-s, \cdot) E(s, \cdot) d s, \varphi\right\rangle
$$




$$
\begin{aligned}
& +\langle\beta f(E), \varphi\rangle+\left\langle\int_{0}^{t} \alpha(t-s, \cdot) f(E(s, \cdot)) d s, \varphi\right\rangle+\left\langle\hat{\gamma} \frac{d}{d t} f(E), \varphi\right\rangle \\
& +\left\langle c^{2} h^{\prime} E^{\prime}, \varphi^{\prime}\right\rangle+c \dot{E}(t, 0) \varphi(0)=\langle\mathcal{J}(t, \cdot), \varphi\rangle_{V^{*}, V}
\end{aligned}
$$

and

$$
E(0, z)=\Phi(z), \quad \dot{E}(0, z)=\Psi(z)
$$

Using a Galerkin type approach and special considerations for the nonlinear terms we were able to show [14] that, under fairly general assumptions on the nonlinearity $f$, a unique global weak solution exists and it depends continuously on initial data.

Thus the one dimensional problem with nonlinearly forced dynamics for the polarization is well-posed. This system can also be thought of as a type of approximation (using truncated Taylor expansions) to the nonlinear polarization dynamics:

$$
\dot{\vec{P}}+f(\vec{P})=k \vec{E}
$$

and

$$
\ddot{\vec{P}}+\gamma \dot{\vec{P}}+f(\vec{P})=k \vec{E}
$$

which represent nonlinear Debye and Lorentz mechanisms and are suggested in [18]. Currently a study is underway to compare these different systems theoretically and computationally.

\subsection{Extension to higher dimensions}

To extend the above methodology to more realistic situations one needs to formulate the problems in higher (two or three) dimensions and demonstrate the applicability of the variational framework in that setting. The work on microwave interrogating signals has been extended to two dimensions computationally [7] for a diagonally anisotropic slab of material. The extensions to higher dimensions and higher frequencies are closely related and several new challenges arise.

Theoretically, the one-dimensional model formulated above depends on the tacit assumption that the polarization field $\vec{P}$ in the dielectric remains parallel to the electric field $\vec{E}$. Even then, the usual Maxwell equation $\nabla \cdot \vec{D}=0$ along with the constitutive law $\vec{D}=\epsilon_{0} \epsilon_{r} \vec{E}+f_{1}(\vec{P}) \vec{P}$ need not result in $\nabla \cdot \vec{E}=0$. This is important in deriving the second order form of Maxwell's equation where the identity $\nabla \times \nabla \times \vec{E}=\nabla(\nabla \cdot \vec{E})-\nabla^{2} \vec{E}$ results in the simple Laplacian only if $\nabla \cdot \vec{E}=0$ or if one assumes this term is negligible as often done in nonlinear optics ([18], p. 54-60). We believe that it may be important to consider the full system to capture the dynamics of the propagated electromagnetic signal.

Experimentally it is known that birefringence occurs in anisotropic dielectrics as a result of the different phase velocities for different directions of the electric field polarization. 
Birefringence can also occur without anisotropy when two different modes of the electric field are coupled nonlinearly. It is present in living organisms even at microwave frequencies, but its effect is small at 1-3 GHz. At frequencies higher than $10 \mathrm{GHz}$ the effect cannot be neglected and anisotropy needs to be taken into account even if linear polarization dynamics are assumed. Anisotropic effects and the tensor nature of the dielectric constant is especially important for the detection of aerosols, suspended particles in fluids, and bacteria (e.g., anthrax) with membranes of complex geometries. At even higher frequencies where nonlinearities in the polarization dynamics become pronounced, it is expected that there are strong interactions between the nonlinear and anisotropic effects, so their correct modeling is crucial for the accurate representation of the propagation and reflection dynamics.

In the computational treatment of the two- or three-dimensional interrogation problem one encounters several difficulties. Naturally, the higher spatial dimension involves increased computational complexities, especially with nonlinear polarization dynamics. However, there are additional inherent challenges. As described in [7], the interrogating signals from a finite antenna produce oblique incident waves on a planar medium, and they must be treated in reflections as well. Thus one cannot use the uniformity assumption as in the one-dimensional model to reduce the problem to a finite computational domain. In this case the infinite spatial domain must be approximated by a finite computational domain with artificial boundaries. At these boundaries some type of boundary damping must be employed to remove unwanted numerical reflections. In [7] perfectly matched layers (PML-s) along with Enquist-Majda absorbing boundary conditions are used to successfully control these reflections. Another possibility that is currently being explored is to enlarge the computational domain so that reflections from the sample and from the artificial boundaries might be separated in time.

In summary, we believe that the variational framework for the interrogation problem is suitable for capturing important dynamic effects associated with the propagation of electromagnetic pulses in different materials. Although it is challenging both theoretically and computationally, it has a great potential for providing a firm foundation for novel imaging methods which can contribute to the current efforts for greater security against terrorist activities.

\section{Concluding Remarks}

The atmosphere of the real threat of terrorism at home and abroad has unfortunately initiated a new environment and urgency for scientific and technological research. While some in our community suggest [19] "for the most part we do not need new methods," our view is somewhat different. While it is true that we in the mathematical sciences community have techniques and approaches that may be extremely important in the new problems arising in the war on bioterrorism, as we enjoin this fight we will find much work to do to pursue our ideas in a relevant manner. It is not true that we have all the tools we need nor are those we do have in the needed form for immediate application. Our strong belief is that more will be required of mathematics and statistics than collecting of existing tools and applying them with relatively straightforward modifications. 
The focus in this chapter is quite narrow and the actual effort on terrorism requires a multidisciplinary as well as interdisciplinary approach beyond that of this volume and beyond that which the community has embraced to date. There is a virtual catalogue of far ranging topics from the engineering, physical, mathematical and biological sciences: data mining, network analysis, biomathematics, genomics, operations research (game theory, risk analysis, logistics), etc., which must be combined with the social and psychological sciences (individual and group behavior, e.g., fanaticism, cognition, etc.) in ways and on a scale unprecedented in the history of science. And this must be done with a new sense of urgency. For example, the development of agent-specific biosensors, sometimes in the context of "smart" materials, has for some time been a priority at several of our national labs; the needs have been heightened by events of the past several years.

Lest our view appear too pessimistic, we hasten to add that while we do not have ready "solutions" to questions and problems that perhaps are only now being precisely formulated, the mathematical and statistical sciences do have a rich history of model development with associated tools and techniques. This will undoubtedly provide a solid foundation that will prove extremely valuable in the pursuit of many specific problems related to terrorism in general and bioterrorism in particular. We are optimistic about the value we can bring to society in this essential effort.

\section{Acknowledgments}

Research reported here was supported in part by the U.S. Air Force Office of Scientific Research under grant AFOSR F49620-01-1-0026 and in part by the Joint DMS/NIGMS Initiative to Support Reserach in the Area of Mathematical Biology under grant 1R01GM67299-01.

The authors are grateful to Dr. Richard Albanese, Dr. Carlos Castillo-Chavez and Dr. Marie Davidian for several informative discussions. Part of this chapter was completed while H.T.B. was a visitor to the Mittag Leffler Institute of the Royal Swedish Academy of Sciences, Djursholm, Sweden. Collaboration was also facilitated while all authors were visitors to the Statistical and Applied Mathematical Sciences Institute (SAMSI), Research Triangle Park, $\mathrm{NC}$.

\section{References}

[1] R. A. Albanese, H. T. Banks, M. V. Evans, and L. K. Potter, Physiologically based pharmacokinetic models for the transport of trichloroethylene in adipose tissue, Bulletin of Mathematical Biology, 64 (2002), pp. 97-131.

[2] R. A. Albanese, H. T. Banks, And J. K. RAYe, Non-destructive evaluation of materials using pulsed microwave interrogating signals and acoustic wave induced reflections, Tech. Rep. CRSC-TR02-15, Center for Research in Scientific Computation, 
North Carolina State University, April 2002; Inverse Problems, 18 (2002), pp. 1935 1958.

[3] C. A. Balanis, Advanced Engineering Electromagnetics, John Wiley \& Sons, Inc., New York, 1989.

[4] H. T. BANKs, Identification of nonlinear delay systems using spline methods, in Nonlinear Phenomena in Mathematical Sciences, V. Lakshmikantham, ed., Academic Press, Inc., New York, 1982, pp. $47-55$.

[5] H. T. BANKS AND K. L. BIHARI, Modeling and estimating uncertainty in parameter estimation, Inverse Problems, 17 (2001), pp. 1-17.

[6] H. T. Banks, D. M. Bortz, And S. E. Holte, Incorporation of variability into the mathematical modeling of viral delays in HIV infection dynamics, Tech. Rep. CRSCTR01-25, Center for Research in Scientific Computation, North Carolina State University, September 2001; Mathematical Biosciences, to appear.

[7] H. T. Banks And B. L. Browning, Time domain electromagnetic scattering using perfectly matched layers, Tech. Rep. CRSC-TR02-24, Center for Research in Scientific Computation, North Carolina State University, July 2002.

[8] H. T. Banks, M. W. Buksas, And T. Lin, Electromagnetic Material Interrogation Using Conductive Interfaces and Acoustic Wavefronts, SIAM Frontiers in Applied Mathematics, Philadelphia, 2000.

[9] H. T. Banks And J. A. BuRns, Hereditary control problems: Numerical methods based on averaging approximations, SIAM Journal of Control and Optimization, 16 (1978), pp. $169-208$.

[10] H. T. BANKS AND F. KAPPEL, Spline approximations for functional differential equations, Journal of Differential Equations, 34 (1979), pp. $496-522$.

[11] H. T. Banks AND F. KoJIma, Boundary shape identification problems in two dimensional domains related to thermal testing of materials, Quart. Appl. Math, 47 (1989), pp. $273-293$.

[12] H. T. Banks, F. Kojima, And W. P. Winfree, Boundary estimation problems arising in thermal tomography, Inverse Problems, 6 (1990), pp. 897 - 921.

[13] H. T. Banks And K. Kunisch, Estimation Techniques for Distributed Parameter Systems, Birkhauser, Boston, 1989.

[14] H. T. Banks AND G. A. Pinter, Maxwell-systems with nonlinear polarization, Nonlinear Analysis, to appear. 
[15] H. T. Banks And L. K. Potter, Probabilistic methods for addressing uncertainty and variability in biological models: Application to a toxicokinetic model, Tech. Rep. CRSC-TR02-27, Center for Research in Scientific Computation, North Carolina State University, September 2002; Mathematical Biosciences, submitted.

[16] P. Billingsley, Convergence of Probability Measures, Wiley, New York, 1968.

[17] D. M. Bortz, Modeling, analysis and estimation of an in vitro HIV infection using functional differential equations, $\mathrm{PhD}$ thesis, North Carolina State University, Raleigh, NC, August 2002.

[18] R. W. Boyd, Nonlinear Optics, Academic Press, San Diego, 1992.

[19] C. Castillo-Chavez and F. S. Roberts, eds., Report on DIMACS Working Group Meeting: Mathematical Sciences Methods for the Study of Deliberate Releases of Biological Agents and their Consequences, Preliminary Draft, April 2002.

[20] M. Davidian and D. Giltinan, Nonlinear Models for Repeated Measurement Data, Chapman and Hall, London, 1998.

[21] R. S. ElLiot, Electromagnetics: History, Theory and Applications, IEEE Press, New York, 1993.

[22] B. G. Lindsay, Mixture Models: Theory, Geometry and Applications, vol. 5 of NSFCBMS Regional Conf. Series in Probability and Statistics, Inst. Math. Stat., Haywood, CA, 1995.

[23] J. L. Lions, Optimal Control of Systems Governed by Partial Differential Equations, Springer-Verlag, New York, 1971.

[24] M. A. Medinsky and C. D. Klahssen, Toxicokinetics, in Casarett and Doull's Toxicology: The Basic Science of Poisons, McGraw-Hill, Health Professions Division, New York, 5th ed., 1996.

[25] D. M. Mittleman, M. Gupta, R. Neelamani, R. G. Baraniuk, J. V. Rudd, AND M. Koch, Recent advances in terahertz imaging, Applied Physics B, 68 (1999), pp. $1085-1094$.

[26] O. Pironneau, Optimal Shape Design for Elliptic Systems, Springer-Verlag, New York, 1983.

[27] L. K. Pотter, Physiologically based pharmacokinetic models for the systemic transport of trichloroethylene, PhD thesis, North Carolina State University, Raleigh, NC, August 2001. 
[28] M. S. Roberts And M. Rowland, A dispersion model of hepatic elimination: 1. Formulation of the model and bolus considerations, Journal of Pharmacokinetics and Biopharmaceutics, 14 (1986), pp. 227-260.

[29] M. E. Rogel, L. I. Wu, And M. Emerman, The human immunodeficiency virus type 1 vpr gene prevents cell proliferation during chronic infection, Journal of Virology, 69 (1995), pp. $882-888$.

[30] B. K. P. Scaife, Principles of Dielectrics, Clarendon Press, Oxford, 1989.

[31] C. Sulem And P. L. Sulem, The Nonlinear Schrodinger Equation, Self-Focusing and Wave Collapse, Springer-Verlag, New York, 1999.

[32] S.Wang, B. Ferguson, C. Mannella, D. Gray, D. Abbott, and X. C. Zhang, Powder detection using THz imaging, in OSA Technical Digest, Postconference Edition, Optical Society of America, Washington, D.C., 2002, p. 132.

[33] J. WlokA, Partial Differential Equations, Cambridge Univ. Press, 1992. 